\title{
Measuring Limb Volume: Accuracy and Reliability of Tape Measurement Versus Perometer Measurement
}

\author{
Amy R. Sharkey, BMBCh, Samuel W. King, BMBCh, Rachel Y. Kuo, BMBCh, Shixin B. Bickerton, MRCS, \\ Alexander J. Ramsden, FRCS (Plast), ${ }^{3,4}$ and Dominic Furniss, FRCS (Plast) ${ }^{2-4}$
}

\begin{abstract}
Background: Accurate limb volume measurement is key in the assessment of outcomes in lymphedema microsurgery. There are two commonly used methods as follows: manual circumferential measurement (tape) or Perometer measurement. There are no data on the intra- and interclass correlation of either method, making it difficult to establish a gold standard of limb volume measurement. We aim to assess the intra- and interclass correlation of each method to establish the most appropriate method for clinical practice and future research studies, aiming to compare the accuracy and reliability of tape measurement as assessed against Perometer measurement. Methods and Results: Student volunteers and experts (lymphedema practitioners) were each asked to perform repeat tape and Perometer measurements on the upper or lower limb of one healthy volunteer. Perometer measurements were globally more accurate than tape (average SE [Perometer]: 23.23 vs. 77.21 [tape]). For intraobserver reliability, experts outperformed students in all domains tested, with little difference in intraobserver reliability using tape or Perometer (average Cronbach's alpha 0.9597 [expert)] vs. 0.6033 [student]). Conclusions: We recommend that, for increased interobserver reliability, the Perometer provides a more reliable standard of limb volume measurement.
\end{abstract}

Keywords: lymphedema, lymphoedema, Perometer, limb volume

\section{Introduction}

$\mathbf{L}$ YMPHEDEMA IS A chronic condition of localized fluid retention and tissue swelling, caused by a compromised lymphatic system. Lymphedema has historically been broadly categorized into two subsets as follows: primary lymphedema, caused by a fault in lymphatic system development, ${ }^{1}$ and secondary lymphedema, caused by damage to the lymphatic system, commonly due to treatment of cancer, infection, or trauma. ${ }^{2}$ Secondary lymphedema is commonly caused by axillary dissection and/or radiotherapy, received as part of the treatment regime for breast cancer in $15 \%-20 \%$ of women. ${ }^{3}$ This classification is no longer straightforward, as more recent research suggests that some apparently secondary lymphedemas may have an underlying primary component. ${ }^{4,5}$ Regardless of origin, lymphedema has an estimated prevalence of
1.33-3.99 per 1000 population $^{6,7}$ and is now thought to affect around 200,000 people in the United Kingdom. ${ }^{8}$

The increasing use of microsurgical techniques, such as lymphaticovenular anastomosis ${ }^{9}$ and vascularized lymph node transfer, ${ }^{10}$ to treat lymphedema has highlighted the importance of accurate limb volume measurement. Without an accurate assessment of volume, it is difficult to assess the impact of these microsurgical treatments and to monitor their longevity. The lack of clarity on this topic is becoming an increasing problem due to the increasing prevalence of lymphedema. ${ }^{11}$ Establishing a gold standard of limb volume measurement is key for assessing the outcome of lymphedema treatment. ${ }^{12}$

The difficulties in measuring limb volume are demonstrated by the number of techniques available: circumferential measurement (at predetermined short intervals along the

\footnotetext{
${ }^{1}$ John Radcliffe Hospital, Oxford University Medical School, John Radcliffe Hospital, Oxford, United Kingdom.

${ }^{2}$ Nuffield Department of Orthopaedics Rheumatology and Musculoskeletal Sciences, Botnar Research Centre, Oxford University, Oxford, United Kingdom.

${ }^{3}$ Department of Plastic and Reconstructive Surgery, Nuffield Orthopaedic Centre, Oxford, United Kingdom.

${ }^{4}$ Oxford Lymphoedema Practice, The Manor Hospital, Oxford, United Kingdom.
} 
limb, with these measurements being used to calculate volume), optoelectric limb volumetry (Perometer), computer tomography, 3D laser scanning, and various methods of water displacement. ${ }^{13}$ It is difficult to know, at present, which measurement is the most accurate. Early work on this subject has validated the Perometer, ${ }^{14}$ suggesting that it is a reliable and convenient tool for the measurement of limb volume.

Discrepancies in measurement methods are frequently cited as a significant limitation when comparing data from multiple studies, ${ }^{15-17}$ hindering evidence based progression in lymphedema management. Although various methods of measuring limb volume have been described which correlate with self-reported symptoms, ${ }^{18}$ the lack of agreement between the methods establishes that they are not interchangeable. ${ }^{19}$ This study aims to compare the accuracy of tape measurement as assessed against Perometer measurement, as the two most widely used and widely available methods of measuring limb volume. We aim to assess the intra- and interclass correlation of both methods.

\section{Materials and Methods}

Ethical approval for this study was gained from the University of Oxford Central University Research Ethics Committee (approval number MS-IDREC-C1-2015-124).

\section{Tape measurements}

We use circumferential tape measurements to calculate limb volume. Modeling the limb as a cylinder, we used the equation $\pi r^{2} h$, where the radius was measured every $4 \mathrm{~cm}$ to take into account the variation in limb radius, and the final calculation was a composite of these measurements. Both limbs were in a constant position (horizontal for arm measurements and vertical for leg measurements) for all measurements.

\section{The Perometer}

The Perometer takes measurements every $4.7 \mathrm{~mm}$ and uses these to calculate volume.

\section{Student measurements}

Videos were made showing how to use the Perometer and how to perform circumferential tape measurements every $4 \mathrm{~cm}$ from the ankle to the upper thigh. Medical student volunteers watched these videos and then carried out both Perometer and circumferential measurements on either the right arm or right leg of one healthy subject. The subject was seated between measurements, and all measurements were carried out in the same position (horizontally for arms and vertically for legs). All measurements were repeated once to assess intraobserver reliability.

\section{Expert measurements}

To compare the data from lymphedema practitioners (experts), we repeated the above set up at the British Lymphoedema Society (BLS) conference in October 2015. To participate in this study, all "experts" had to be experienced lymphedema practitioners who were members of the BLS. Rather than showing a video, practitioners used the Perometer and performed circumferential tape measurements using their usual method. All practitioners were experienced in both methods. We asked all experts to return to perform a second measurement, to compare the expert measurements with the student measurements and to assess differences in intraobserver reliability between experts and students.

\section{Data analysis}

To standardize both data sets, the minimum limb length measurement recorded was taken as a base, and all further measurements above the minimum were excluded so that each measurement started and ended at the same point. To do this, the minimum limb length recorded on the Perometer for both the experts and students was found, and all other Perometer data were standardized to this measurement, using the proprietary PeroPlus software (Pero-System Messgeräte $\mathrm{GmbH}$, Wuppertal, Germany). The minimum length of arm or leg for circumferential measurements was also found and volume calculations did not include measurements exceeding this minimum length. This allowed direct comparison of data between all measurers.

For interobserver reliability, we calculated the mean and 95\% confidence intervals (CIs). For intraobserver reliability we used Cronbach's alpha, which measures correlation between measurements. This is reported with two statistical measures: first, the average inter-item coefficient (IC) and second the scale reliability coefficient, reported as alpha. This was calculated after excluding missing repeat values. Generally speaking, an alpha value of $0.7-0.8$ is regarded as showing acceptable internal consistency. Good consistency is $0.8-0.9$ and $>0.9$ is excellent.

\section{Results}

For the student data, 23 repeated arm measurements and 22 repeated leg measurements were collected, for both tape and Perometer. For the expert tape data, there were 12 arm measurements, 2 of which were repeated, and 14 leg measurements, 6 of which were repeated. For the expert Perometer data, there were 12 arm measurements, 2 of which were repeated, and 13 leg measurements, 4 of which were repeated.

\section{Tape versus Perometer accuracy}

Tables 1 and 2 compare the accuracy of measurements using the tape and Perometer, respectively. Graphic representation is shown in Figure 1.

Considering tape measurement of arms, the standard error (SE) was lower in the expert measurements than in the students (average SE 25 [expert] vs. 29.55 [student]), and the expert's CI was narrower. With leg measurements, the SE and CIs were much wider in general, with the students having significantly lower SEs (average SE 63.75 [student] vs. 164.43 [expert]). This is most likely because we asked the practitioners to use their usual method of measurement with tape, rather than teaching a set method as we did with the students.

Compared to tape measurements, the Perometer measurements had, on average, lower SEs and narrower CIs when used by both students and experts (average SE arm measurements [experts and students] 24.88 [Perometer] vs. 28.04 [tape]) average SE leg measurements [experts and students] 
Table 1. Interrater Reliability of Limb Volume Measurements Using Tape Measure

\begin{tabular}{|c|c|c|c|c|c|}
\hline $\begin{array}{l}\text { Volunteer } \\
\text { Measurement No. }\end{array}$ & Limb & $\mathrm{N}$ & Mean & $95 \% C I$ & $S E$ \\
\hline Student & Arm & & & & \\
\hline Tape 1 & & 23 & 3377.09 & $3312.20-3441.98$ & 31.29 \\
\hline Tape 2 & & 23 & 3364.43 & $3306.74-3422.12$ & 27.82 \\
\hline \multicolumn{6}{|l|}{ Practitioner } \\
\hline Tape 1 & & 12 & 3433.53 & $3291.05-3401.11$ & 25.00 \\
\hline Tape $2^{\mathrm{a}}$ & & 2 & & & \\
\hline Student & Leg & & & & \\
\hline Tape 1 & & 22 & 9267.49 & $9116.12-9418.87$ & 72.79 \\
\hline Tape 2 & & 22 & 9301.50 & 9187.67-9415.34 & 54.74 \\
\hline \multicolumn{6}{|l|}{ Practitioner } \\
\hline Tape 1 & & 14 & 10187.64 & $9799.74-10575.54$ & 179.55 \\
\hline Tape 2 & & 6 & 9873.06 & $9489.28-10256.84$ & 149.30 \\
\hline
\end{tabular}

${ }^{a}$ Note for practitioner measurements of arm with tape there were only two repeat readings so statistical analysis has not been performed. $\mathrm{CI}$, confidence interval; SE, standard error.

22 [Perometer] vs. 114.10 [tape]). The only measurement not to follow this statement was expert measurements of arms, in which the SE of tape measurement was lower than the Perometer reading (SE 25 vs. 38.99). Using the Perometer, experts had higher standard error than students in both arms and legs, which is likely an anomaly due to the significantly lower numbers of expert readings due to time constraints at the BLS conference.

\section{Intraobserver reliability}

Table 3 reports intraobserver reliability. We asked experts to use their usual method of tape measurement, rather than teaching a set method as we did with the students, to preserve their expertise; by not using their usual technique they would likely have lost a degree of expertise and likely would have erroneously decreased their intraobserver reliability. As such, the expert's intrarater reliability is significantly better than students.

As previously mentioned, there were no missing values for the student data, hence the unusually low alpha value for the student's leg measurements. This suggests that there is ac- ceptable internal consistency with manual measurements of legs. The expert data show excellent internal consistency when using the Perometer and tape to measure legs (Cronbach's alpha of 0.9009 and 0.9442 , respectively), suggesting that expertise with either equipment may be the key to consistency.

As Cronbach's alpha is unreliable with missing data, we have excluded all measurements that were not repeated. Using only the repeated data, there is excellent intraobserver reliability in experts using both tape and Perometer measurements. However, the number of repeat expert readings was low, limiting the interpretation of intraobserver reliability data for experts.

\section{Discussion}

In terms of accuracy, on average the SE was smaller and CIs were narrower with the Perometer measurements, suggesting that it is broadly more accurate than tape measurement.

For intraobserver reliability, Cronbach's alpha was $>0.9$ in all "expert" domains, showing excellent intraobserver reliability.

Table 2. Interrater Reliability of Limb Volume Measurements Using a Perometer

\begin{tabular}{|c|c|c|c|c|c|}
\hline $\begin{array}{l}\text { Volunteer } \\
\text { Measurement No. }\end{array}$ & Limb & $\mathrm{N}$ & Mean & $95 \% C I$ & $S E$ \\
\hline Student & Arm & & & & \\
\hline Perometer 1 & & 23 & 3299.91 & $3259.44-3340.39$ & 19.52 \\
\hline Perometer 2 & & 23 & 3276.61 & $3243.18-3310.04$ & 16.12 \\
\hline \multicolumn{6}{|l|}{ Practitioner } \\
\hline Perometer 1 & & 12 & 3346.08 & $3349.29-3517.74$ & 38.99 \\
\hline Perometer $2^{\mathrm{a}}$ & & 2 & & & \\
\hline Student & Leg & & & & \\
\hline Perometer 1 & & 22 & 9606.27 & $9573.86-9638.69$ & 15.59 \\
\hline Perometer 2 & & 22 & 9537.68 & $9514.40-9560.97$ & 11.20 \\
\hline \multicolumn{6}{|l|}{ Practitioner } \\
\hline Perometer 1 & & 13 & 9831.23 & $9771.14-9891.32$ & 27.58 \\
\hline Perometer 2 & & 4 & 9818.25 & $9711.27-9925.23$ & 33.62 \\
\hline
\end{tabular}

\footnotetext{
${ }^{a}$ Note for practitioner measurements of arm with Perometer there were only two repeat readings so statistical analysis has not been performed.
} 

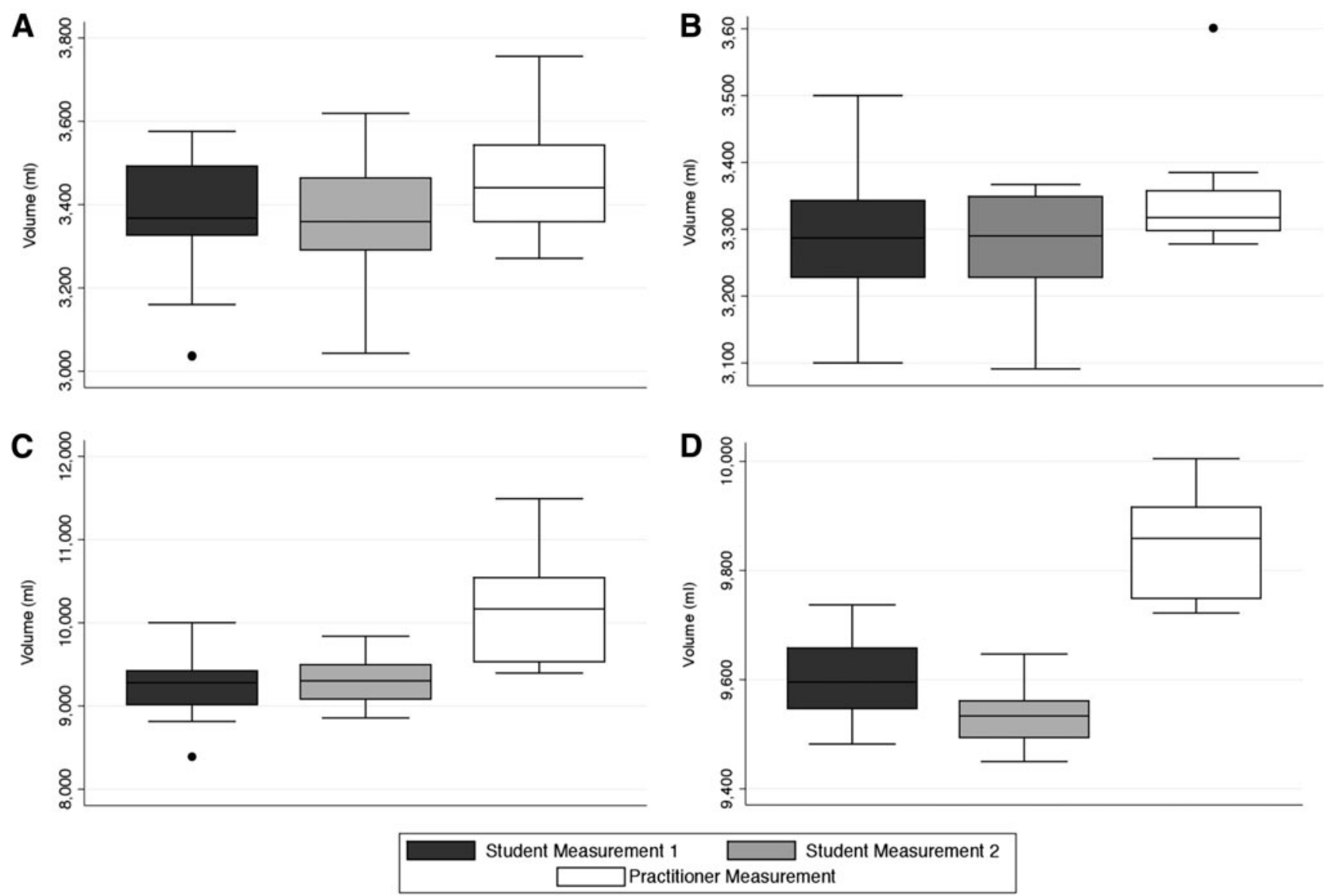

FIG. 1. Box plots showing volume measurements for students and practitioners. In students the second measurement is shown for comparison; with practitioners the number of repeats was too low to allow comparison.(A) Arm measurements using tape.(B) Arm measurements using the Perometer.(C) Leg measurements using tape.(D) Leg measurements using the Perometer. • indicates anomalous measurements.

This is as one might expect for trained practitioners. However, a key limitation of this study is the low number of repeats in the expert group. With student data, the numbers are higher and the results much more variable. The student Perometer measurement of legs showed very low internal consistency, which needs further validation.

For interobserver reliability, the experts outperformed the students in all domains tested except tape measurement of

\section{Table 3. Intrarater Reliability of Limb Volume Measurements with Tape and Perometer}

\begin{tabular}{lccc}
\hline Measurement method & Limb & $\begin{array}{c}\text { Scale } \\
\text { Inter-item } \\
\text { covariance }\end{array}$ & $\begin{array}{c}\text { reliability } \\
\text { coefficient } \\
\text { (alpha) }\end{array}$ \\
\hline Student, tape & Arm & 10280.42 & 0.6755 \\
Practitioner, tape & & 2221.21 & 0.9947 \\
Student, Perometer & & 4545.96 & 0.7635 \\
Practitioner, Perometer & Leg & 5136 & 0.9991 \\
Student, tape & & 136054.2 & 0.7210 \\
Practitioner, tape & 587.0909 & 0.9442 \\
Student, Perometer & & 4330.167 & 0.9009 \\
Practitioner, Perometer & & & \\
\hline
\end{tabular}

legs. This is likely because we invited the experts to use their normal methods of measurement; by preserving their expertise (and therefore their intraobserver reliability) we adversely affected their interobserver reliability in measurements in which margins of error were larger (i.e., circumferential measurement of legs) as they tended to use different starting points to measure from and different techniques for measuring.

Overall, for interobserver reliability, the Perometer appears consistently better than tape measurement. This is a relevant finding, as patients visiting a lymphedema clinic may have their affected $\operatorname{limb}(\mathrm{s})$ measured by a different lymphedema practitioner each time. Increasing interobserver reliability using a Perometer would allow more accurate monitoring of the effect of treatment. In addition, the Perometer is universally faster than tape measurement, thus could save a significant amount of clinic time. Although we did not aim to time measurements, we estimate that the Perometer took 2 minutes per limb and tape measurement $\sim 10$ minutes. However, there is significant monetary cost attached to the Perometer, and while it has a function to automatically prescribe garments from the measurements it takes, thus saving further time and increasing the utility of the device, it is not universally available. As intraobserver reliability was high in the expert group for tape and Perometer measurements 
alike, if a Perometer was not available, using the same trained practitioner for each patient's measurements would also be a reproducible way of assessing treatment outcomes. However, as human factors make this difficult to achieve in a clinic environment, a more accurate and reliable way to monitor clinical response would be the consistent use of the Perometer.

\section{Conclusion}

The ability to accurately measure limb volume is increasingly relevant given the increasing prevalence of lymphedema and the variety of microsurgical reconstructive techniques available. The gold standard treatment of lymphedema is controversial. To assess the merits of each treatment in a reliable manner, a reliable way of measuring limb volume must be established.

It is beyond the scope of this study to state a definitive gold standard technique for limb volume measurement, as it compares only the two most widely used and available methods. However, the Perometer gives more accurate results overall and is much quicker to use. Further work is required, with increased numbers of participants and comparison with other commonly used methods of limb volume measurement.

\section{Author Disclosure Statement}

No competing financial interests exist.

\section{References}

1. Connell F, et al. A new classification system for primary lymphatic dysplasias based on phenotype. Clin Genet 2010; $77: 438-452$.

2. Warren AG, Brorson H, Borud LJ, Slavin S. A. Lymphedema: A comprehensive review. Ann Plast Surg 2007; 59: 464-472.

3. Clark B, Sitzia J, Harlow W. Incidence and risk of arm oedema following treatment for breast cancer: A three-year follow-up study. QJM 2005; 98:343-348.

4. Rockson SG. Secondary lymphedema: Is it a primary disease? Lymphat Res Biol 2008; 6:63-64.

5. Rockson SG. The unique biology of lymphatic edema. Lymphat Res Biol 2009; 7:97-100.

6. Moffatt CJ, et al. Lymphoedema: An underestimated health problem. QJM 2003; 96:731-738.

7. Lymphoedema Support Network. Available at: www .publications.parliament.uk/pa/cm201415/cmselect/cmhealth/ 401/401vw09.htm Last accessed December 16, 2016.

8. National Health Service United Kingdom. Lymphoedema, 13 November 2016. Available at: www.nhs.uk/conditions/ Lymphoedema Last accessed December 16, 2016.

9. Campisi C, et al. Microsurgery for lymphedema: Clinical research and long-term results. Microsurgery 2010; 30: 256-260.
10. Cheng $\mathrm{M}-\mathrm{H}$, et al. Vascularized groin lymph node flap transfer for postmastectomy upper limb lymphedema: Flap anatomy, recipient sites, and outcomes. Plast Reconstr Surg 2013; 131:1286-1298.

11. DiSipio T, Rye S, Newman B, Hayes S. Incidence of unilateral arm lymphoedema after breast cancer: A systematic review and meta-analysis. Lancet Oncol 2013; 14:500-515.

12. Best practice for the management of lymphoedema. International consensus. London: MEP Ltd, 2006.

13. Rabe E, Stücker M, Ottillinger B. Water displacement leg volumetry in clinical studies - a discussion of error sources. BMC Med Res Methodol 2010; 10:5.

14. Stanton AW, Northfield JW, Holroyd B, Mortimer PS, Levick JR. Validation of an optoelectronic limb volumeter (Perometer). Lymphology 1997; 30:77-97.

15. Hayes S, Janda M, Cornish B, Battistutta D, Newman B. Lymphedema secondary to breast cancer: How choice of measure influences diagnosis, prevalence, and identifiable risk factors. Lymphology 2008; 41:18-28.

16. Cormier JN, Xing Y, Zaniletti I, Askew RL, Stewart BR. Minimal limb volume change has a significant impact on breast cancer survivors. Lymphology 2009; 42:161-175.

17. Armer JM, Stewart BR, Shook RP. 30-month post-breast cancer treatment lymphoedema. J Lymphoedema 2009; 4: $14-18$.

18. Ridner SH, Montgomery LD, Hepworth JT, Stewart BR, Armer JM. Comparison of upper limb volume measurement techniques and arm symptoms between healthy volunteers and individuals with known lymphedema. Lymphology 2013; 40:35-46.

19. Adriaenssens N, Buyl R, Lievens P, Fontaine C, Lamote J. Comparative study between mobile infrared optoelectronic volumetry with a Perometer and two commonly used methods for the evaluation of arm volume in patients with breast cancer related lymphedema of the arm. Lymphology 2013; 46:132-143.

Address correspondence to: Amy R. Sharkey, BMBCh Oxford University Medical School John Radcliffe Hospital Oxford OX3 9DU United Kingdom

E-mail: amy.sharkey@doctors.org.uk

Dominic Furniss, FRCS (Plast)

Nuffield Department of Orthopaedics Rheumatology and Musculoskeletal Sciences Botnar Research Centre Oxford University Oxford OX3 7HE01865 227233

E-mail: dominic.furniss@ndorms.ox.ac.uk 